\title{
Viewpoint
}

\section{Conjugate vaccines for enteric fever: proceedings of a meeting organized in New Delhi, India in 2009}

\author{
Audino Podda ${ }^{1}$, Allan Saul ${ }^{1}$, Rashmi Arora ${ }^{2}$, Zulfiqar Bhutta ${ }^{3}$, Anju Sinha ${ }^{2}$, Rajni Gaind ${ }^{4}$, Tanu \\ Singhal ${ }^{5}$, Samir Saha ${ }^{6}$, Abdullah Brooks ${ }^{7}$, Laura B. Martin ${ }^{1}$, Yeshwant Amdekar ${ }^{8}$, Amar Jeet \\ Chitkara $^{9}$, Mae Shieh ${ }^{1}$, Ambujam Nair Kapur ${ }^{2}$, Tulsi Das Chugh ${ }^{10}$
}

${ }^{1}$ Novartis Vaccines Institute for Global Health, Siena, Italy, Indian Council of Medical Research, New Delhi, India, ${ }^{3}$ Aga Khan University, Karachi, Pakistan, ${ }^{4}$ VMMC and associated Safdarjung Hospital, New Delhi, India, ${ }^{5}$ Kokilaben Dhirubhai Ambani Hospital, Mumbai, India, ${ }^{6}$ Child Health Research Foundation, Shishu Hospital, Dhaka, Bangladesh, International Centre for Diarrhoeal Disease Research, Bangladesh, ${ }^{8}$ Jaslok Hospital and Research Center, Mumbai, India, ${ }^{9}$ Max Hospital, Pitampura, New Delhi, India, ${ }^{10}$ Indian Academy of National Sciences and BLK Memorial Hospital, New Delhi, India

\begin{abstract}
Enteric fever is responsible for significant morbidity in South Asia and high prevalence of severe disease is seen in children under two years of age. Effective typhoid vaccines are available, but they cannot be used for children under two years of age and also have some limitations in older age groups. Participants supported development of a Salmonella Typhi conjugate vaccine able to induce effective, long-lasting immunity in young children. The role of Salmonella Paratyphi A as a cause of enteric fever was discussed and consensus reached that a bivalent $S$. Typhi-S. Paratyphi A conjugate vaccine is highly desirable; however, considering disease epidemiology and the advanced status of vaccine development, rapid introduction of monovalent $S$. Typhi conjugate vaccine into vaccination programs of South Asia was recommended. Prevention should be emphasized, available vaccines used, and efforts toward improving sanitation continued.

Success of the new vaccine will depend on several factors, including delivery costs and governmental ability to adopt and implement suitable immunization programs. To ensure good immunization coverage, the conjugate vaccine could be administered either to young infants, concomitantly with infant EPI vaccines, or to older infants, concomitantly with measles vaccine, currently given at 9 to 12 months. The need for new combination vaccines, containing both EPI and typhoid antigens, was discussed as a tool to increase coverage and reduce the number of injections and priority conflicts in a crowded infant vaccination schedule. However, stand-alone enteric fever conjugate vaccines would allow more flexibility to immunize different age groups and therefore should be rapidly developed.
\end{abstract}

J Infect Dev Ctries 2010; 4(6):404-411.

(Received 24 March 2010 - Accepted 29 March 2010)

Copyright $(\odot 2010$ Podda et al. This is an open-access article distributed under the Creative Commons Attribution License, which permits unrestricted use, distribution, and reproduction in any medium, provided the original work is properly cited.

\section{Introduction and background}

\section{Scope of the conference}

This two-day meeting, which was organized by the Novartis Vaccines Institute for Global Health, was held on 30 November and 1 December 2009, in New Delhi, under the aegis of the Indian Council of Medical Research (ICMR) and the Dr. B. L. Kapur (BLK) Memorial Hospital of New Delhi to discuss enteric fever in South Asia and the potential of new conjugate vaccines. More specifically, the main objective of the conference was to solicit new data and expert opinions on enteric fever in Bangladesh, India, and Pakistan.

Meeting participants discussed epidemiology and vaccination needs with the aim of facilitating decisions concerning the future health care requirements of the region. Additionally, various options for the development of a vaccine against enteric fever tailored to local public health needs, including preferred vaccine regimes, target age groups, and vaccine formulations and strategies for rapid introduction of new vaccines into national immunization programs were also examined.

\section{Background}

Vaccine-preventable diseases cause around 8 million deaths worldwide per year [1]. According to recent data from the World Health Organization (WHO), infectious diseases predominantly affect low-income populations: $98.6 \%$ of daily adjusted life years (DALYs) and $96.8 \%$ of deaths from infectious disease occur in low- and middle-income countries [1].

Young children are particularly vulnerable; 54\% of DALYs and $43 \%$ of deaths occur in those aged under five years [1]. More specifically, infections 
causing lower respiratory and diarrhoeal diseases are the two main causes of morbidity and mortality in children under five years of age and account for over $50 \%$ of the global disease burden in this age group [1]. However, because of the lack of commercial incentives to invest in new vaccines for pediatric infectious diseases primarily affecting low-income countries, development of effective vaccines specifically targeting these diseases is largely ignored.

The Novartis Vaccines Institute for Global Health (NVGH) aims to facilitate the development of effective and affordable vaccines for neglected infectious diseases in developing countries [2]. NVGH is currently the only not-for-profit vaccine program with direct access to the know-how and resources of a major vaccine manufacturer, Novartis Vaccines \& Diagnostics. Given that diarrhoeal diseases account for over one quarter of infectious diseases in children under five years of age and that enteric fever is one of the main causes of diarrhoeal diseases worldwide, NVGH is actively working on the development of novel conjugate vaccines against this disease [3].

Enteric fever caused by Salmonella Typhi ( $S$. Typhi) or Salmonella Paratyphi A (S. Paratyphi A) is a serious public health concern, especially in Asia, because of its severity and high prevalence. The global burden of enteric fever is estimated to be around 17 million to 21.6 million cases of $S$. Typhi only, with approximately 200,000 to 600,000 deaths every year; about $90 \%$ of cases of enteric fever are recorded in Asia [4]. Currently, health care provision for enteric fever focuses on vaccine prophylaxis (only for typhoid fever, as vaccines against paratyphoid fever are currently not available) and antibiotic treatment. However, effective treatment is significantly hampered by emerging resistance of the causative organisms to many antibiotics. Resistance of $S$. Typhi to chloramphenicol was first observed in 1972 [5]; multi-drug resistant strains appeared in the 1980s, and resistance to fluoroquinolones has increased further since then [6]. Additionally, control of disease by providing clean water and adequate sanitation remains unfortunately a distant objective in many developing countries; therefore development of effective methods for intervention remains an essential priority.

The first-generation vaccine used against $S$. Typhi was a killed whole-cell parenteral vaccine [7]. Although effective after a single dose, this vaccine was very reactogenic and for this reason it has been largely replaced by newer vaccines such as the $\mathrm{Vi}$ polysaccharide vaccine and the live attenuated Ty21a vaccine against $S$. Typhi. These vaccines have been shown to be safe and effective and are recommended by the WHO Strategic Advisory Group of Experts (SAGE) [8]. These vaccines, however, have some relevant limitations; therefore, SAGE also encourages the development of a new generation of better vaccines. In this context, NVGH is working on the development of new polysaccharide-protein conjugate vaccines against both typhoid and paratyphoid fevers which should overcome the limitations of current vaccines [3].

\section{Epidemiology of enteric fever in the Indian Subcontinent \& vaccination needs}

\section{Epidemiology in Pakistan}

There is a high prevalence of enteric fever caused by $S$. Typhi and $S$. Paratyphi $A$ in Pakistan. More than $51 \%$ of pediatric blood culture isolates collected by the Aga Khan University Medical College (AKUMC) emergency services department in Karachi between 1995 and 1999 contained $S$. Typhi or $S$. Paratyphi $A$. $S$. Typhi alone was detected in approximately $43 \%$ of positive cultures (Figure 1).

An age-wise comparison from a communitybased laboratory network of AKUMC (Bhutta et al., 2010 unpublished observations) revealed that enteric fever is broadly a childhood disease: $70 \%$ of all $S$. Typhi cases were in children under 15 years of age, but high incidences were also seen in the 15 to 30 years age groups (Figure 2). Interestingly, a significant number of disease cases were observed in the under 5 years age group, with $S$. Paratyphi predominating in the first year of life.

Figure 1. Spectrum of pediatric blood culture isolates from AKUMC emergency services, Karachi (1995 - 1999)

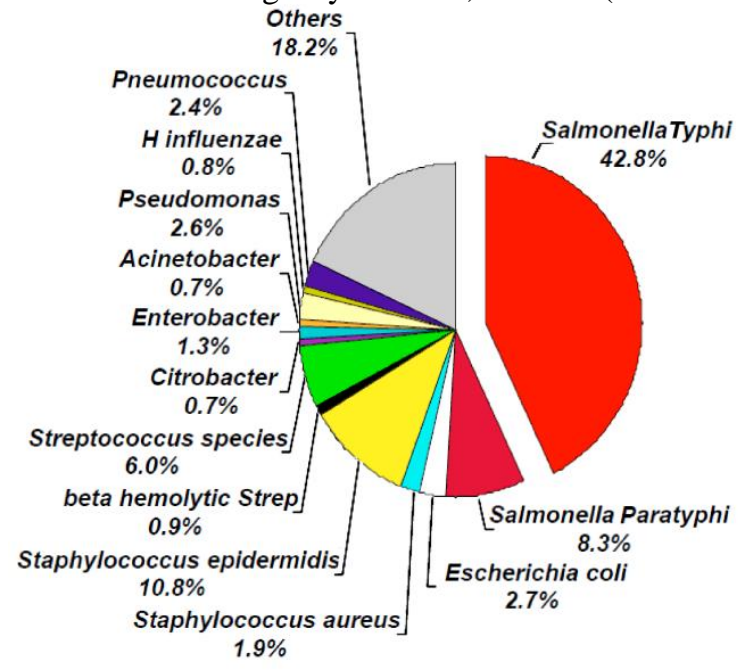


Figure 2. Age distribution of enteric fever in Karachi

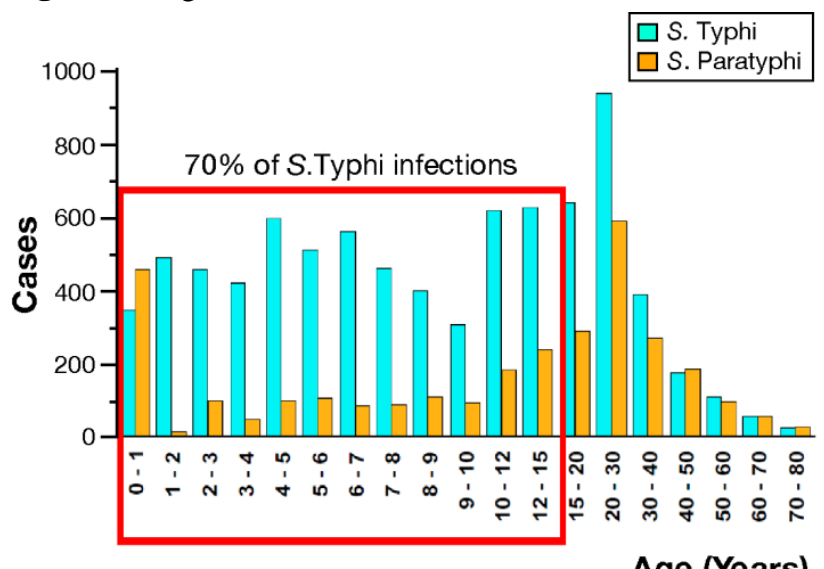

Epidemiology in India

A) Typhoid fever

Despite the limitations of epidemiological data due to the difficulties associated with diagnosing typhoid fever, particularly in children, and the use of passive surveillance studies which result in considerable underreporting, typhoid fever has always been considered a major public health issue in India [9-11]. However, the limitations of the available data further prevented the emergence of a true picture of the magnitude of the problem and suggested that the disease is uncommon in children younger than three years of age. Consequently, typhoid vaccines were mostly assessed in children of school entry age and the optimum age for immunization has remained unclear.

To address the limitations of previous studies based on passive surveillance, a prospective followup study to evaluate the incidence of typhoid fever in preschool children by active surveillance was therefore undertaken. The study further aimed to help define the optimal age of immunization and the vaccine choice [12].

Residents of Kalkaji, Delhi $(\mathrm{N}=8,172)$, were studied from 1 November 1995 to 31 October 1996. The great majority of the study subjects $(\mathrm{N}=7,159)$ were under 40 years of age. There were 1,454 febrile episodes eligible for blood culture, out of which 1,217 were cultured. $S$. Typhi was isolated in 63 cultures. Salmonella strains other than $S$. Typhi were isolated in 24 cultures and other bacteria were detected in 18 cultures. Of the $S$. Typhi culture positives, $16(25 \%)$ were from children under three years of age and 28 (44\%) from children under five years. The overall incidence in patients under 40 years of age was 9.8 culture-confirmed cases per
1,000 person-years, compared to the 2.9 per 1,000 person-years suggested by passive surveillance.

Active surveillance revealed significant typhoid fever morbidity in children less than 5 years of age, with an incidence of 27.3 culture-confirmed cases per 1,000 person-years. Active surveillance of paratyphoid fever revealed an incidence of 3.7 culture-confirmed cases per 1,000 person-years in children less than 5 years of age, while no paratyphoid cases had been detected by passive surveillance. Finally, some cases of paratyphoid fever were detected in infants less than one year of age, while no case of typhoid fever was confirmed in this age group [12].

In conclusion, the findings from this study challenged the previous belief that typhoid fever does not affect preschool children. This study provided, for the first time, data on the age specific incidences of typhoid in younger children (27.3 cases per 1,000 person-years in children under five years of age, with a peak incidence of 51.6 in the third year of life). Of all cases, $44 \%$ occurred in children younger than five years. The belief that disease in children is mild was also contradicted by these results, which supported the need to review vaccination strategies.

The Kalkaji study helped to identify additional relevant information for better characterization of typhoid fever in India. A nested case control study showed that risk factors for typhoid fever include living in nuclear families, eating ice cream, eating meals outside the home, and failure to wash hands before eating. Assessment of antibiotic resistance showed in vivo resistance to ciprofloxacin in nine out of 92 patients, but in general multidrug resistance was not a problem. Finally, the economic evaluation of direct medical and non-medical costs plus indirect costs of the illness indicated that the total cost of enteric fever was 1707 Indian Rupees per case.

More recent population-based prospective surveillance studies were conducted in the Kolkata region to evaluate the typhoid fever disease burden [13]. These studies showed an overall incidence of 242.2 cases per 100,000 person-years and, in addition, that the incidence of typhoid fever in younger children is not significantly different than that in older children and adolescents (340.1 in twoto five-year-old children vs. 495.5 in five- to 15 -yearold children).

B) Paratyphoid fever

Based on data from a global survey in 1995, incidence of $S$. Paratyphi was estimated to be $25 \%$ of 
that of $S$. Typhi (i.e., 0.25 paratyphoid fever illnesses for every typhoid fever illness) [4]. A large community-based study in an urban slum of Delhi confirmed that $S$. Paratyphi A was responsible for approximately $20 \%-25 \%$ of the cases of enteric fever [14]. Several subsequent studies showed a progressive increase in the incidence of paratyphoid fever in India. Among them, a retrospective analysis of the etiology of enteric fever in North India showed an increase in the incidence of S. Paratyphi A cases from $6.5 \%$ to $44.9 \%$ over a five-year period (1994 1998) [15].

Also data from the Vardhman Mahavir Medical College (VMMC) and Safdarjung Hospital, Delhi (Figure 3), indicate that cases of paratyphoid fever are increasing, both in total numbers and in the proportion of cases caused by $S$. Paratyphi $A$. In 2004 , sixty-eight $(30.5 \%)$ out of a total of 223 cases of enteric fever were caused by $S$. Paratyphi $A$.

Another concerning development in $S$. Paratyphi A-caused enteric fever is the growing antibiotic resistance and the subsequent increase in the incidence of complications. Infection in children under five years of age $(p=0.002)$ and infection with nalidixic acid-resistant strains $(\mathrm{p}=0.004)$ were among the major risk factors for complicated disease. Patients less than five years and those infected with nalidixic acid-resistant isolates had 22- and 8-fold higher incidences of complicated disease, respectively [16].

These data, which suggest that the incidence of paratyphoid fever is rising and that there is also a trend for increased drug resistance, reinforce the need to develop an effective vaccine against $S$. Paratyphi A.

Figure 3. Trends in S. Paratyphi A, Safdarjung Hospital (S. Typhi vs. S. Paratyphi A)

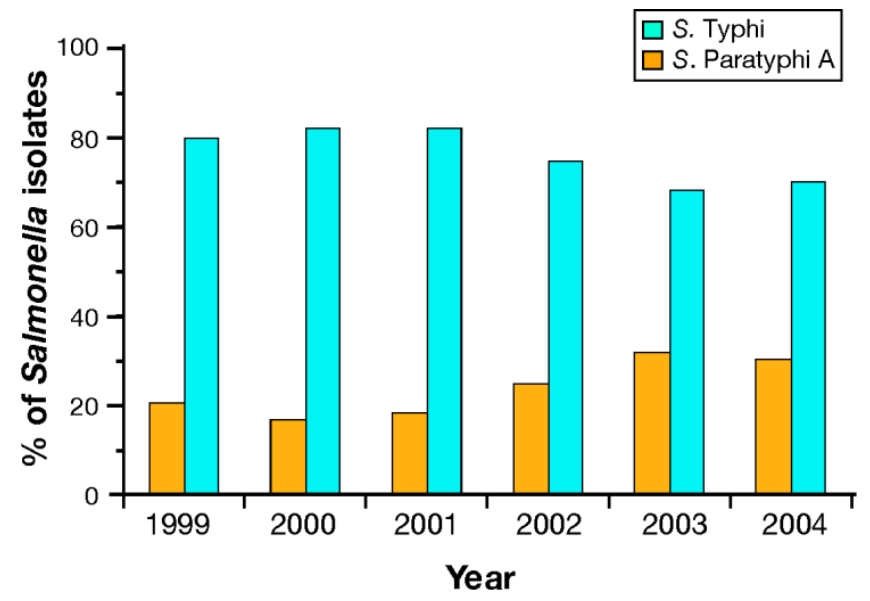

Epidemiology in Bangladesh

With 144 million people, Bangladesh is the seventh most populous country in the world and has a population density of about 1,002 persons/square kilometer, the highest of any other country. Epidemiological data are available from large surveillance programs for invasive bacterial infections performed in different settings.

A multicentre laboratory-based surveillance of community cases in Dhaka (1994 to 2008) in outpatients from higher socioeconomic classes revealed that, out of 63,680 blood cultures done, $5,642(9 \%)$ were positive, and of these, 3,429 (61\%) were positive for $S$. Typhi.

A multicentre hospital-based surveillance for invasive bacterial diseases was performed by a network of seven hospitals (six urban and one rural) in the context of a GAVI supported research project on pneumonia. Children from two to 59 months admitted to the participating hospitals and meeting the case definitions criteria of "pneumonia," "severe pneumonia," and "very severe disease" were enrolled in the study from May 2004 to December 2008. As shown in Figure 4, out of 18,652 blood cultures in six urban hospitals, 495 S. Typhi were isolated (i.e., 34\% of all isolates), while the data from the rural hospital showed a lower rate of isolation and only $26 \%$ of all isolates were $S$. Typhi.

Given the design of the study, all admitted cases were affected by severe disease and therefore the possibility that the number of typhoid and paratyphoid fever cases was underestimated.

A population-based surveillance in rural Bangladesh from April 2006 to March 2008 evaluated children aged $0-59$ months living in a defined catchment area who were referred to the hospital if they had a body temperature $\geq 100.4^{\circ} \mathrm{F}$ for more than three days. Out of 11,439 enrolled subjects, 3,978 were referred to the hospital: $S$. Typhi was the most common isolate from the 3,724 blood cultures.

As shown in Figure 5, drug resistance is increasing. There is a progressive increase in relative resistance to ciprofloxacin, which delays clinical responses and leads to treatment failure or recurrences. Drug resistance also has financial implications: high prevalence of multi-drug resistance and nalidixic acid resistance can lead to hospitalization and a consequent 10 -fold increase in direct costs.

Overall, the prevalence of typhoid fever is higher in urban areas of Bangladesh, as compared to rural 
Figure 4. Multicentre hospital-based surveillance for invasive bacterial diseases in 6 urban hospitals in Bangladesh

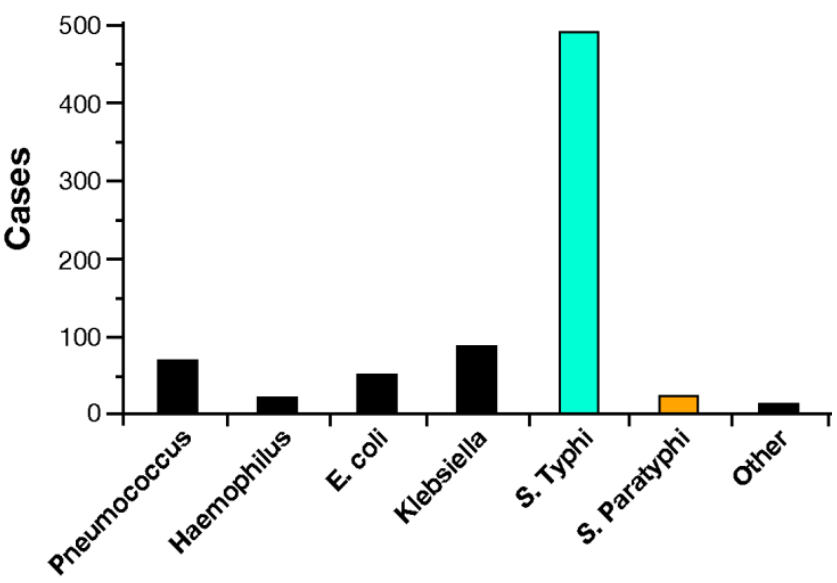

areas, but this picture may change in the near future because of rapid urbanization.

Improved living conditions, including better sanitation, hygiene, and piped water may eventually reduce the incidence of typhoid fever, but these will take a long time to implement in Bangladesh. Meanwhile, immunization with $S$. Typhi vaccines should remain a priority.

Most typhoid fever cases in Bangladesh are in children in their second year of age. This observation was noted in both hospital and community surveys. In addition, the disease severity (as judged by the magnitude of $S$. Typhi bacteremia and duration of hospital stay) is not lower in children younger than 2 years as compared to older children (i.e., older than two years).

Additional data on the epidemiology of enteric fever in Bangladesh have recently been obtained in Kamalapur, a low-income urban community in southeast Dhaka. The overall incidence of typhoid fever from July 2008 to October 2009, across all age groups, was 5.7 episodes $/ 1,000$ person-years while for children under five years of age the incidence was 29.6 episodes $/ 1,000$ person-years. Data from Kamalapur have also shown an increase in the incidence rate of paratyphoid fever, which was overall $13.1 \%$ in a four-year surveillance (2004 to 2009), but $29.7 \%$ in the last 12 months of follow-up. Additionally, the evaluation of the age distribution of $S$. Paratyphi A compared to $S$. Typhi in children less than five years has shown no difference (mean age 32.9 months both for $S$. Typhi and $S$. Paratyphi A).

Data on multi-drug resistance from the same site are shown in Figure 6: 53.9\% of cases were resistant to three drugs ( $\beta$-lactams, chloramphenicol, and
Figure 5. Trends in nalidix acid drug resistance in Bangladesh from 1998-2008 ( $\mathrm{N}=4518)$

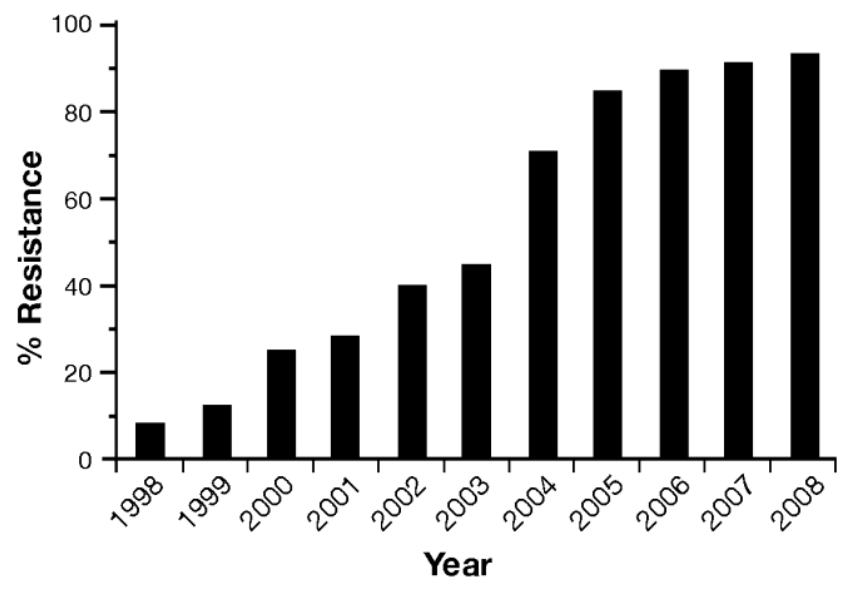

cotrimoxazole) and $51.4 \%$ were resistant to four drugs (including nalidixic acid). Additionally, 81.9\% of cases were intermediately resistant and $0.4 \%$, highly resistant to ciprofloxacin.

\section{Vaccination with currently available vaccines}

As shown in Table 1, Vi polysaccharide and live attenuated Ty21a are the two vaccines against $S$. Typhi currently available. However, both these vaccines have some relevant limitations. They cannot be used in children under two years of age, an age group affected by a significant burden of disease; they induce a short-lived immune response; and, from a practical point of view, they require ad hoc immunization visits (i.e., outside of EPI regular immunization visits), which may cause low vaccination coverage and do increase delivery costs.

Both vaccines are available for use in developing countries and have a proven safety record. Of these two vaccines, the $\mathrm{Vi}$ polysaccharide, a single dose regimen, looks like the more attractive option. To better evaluate the potential use of the $\mathrm{Vi}$ polysaccharide vaccine in global immunization campaigns in endemic countries, Phase IV clusterrandomized effectiveness trials have been recently conducted in Kolkata, India, and Karachi, Pakistan, by the National Institute of Cholera \& Enteric Diseases, Kolkata (India) and the Aga Khan University, Karachi, (Pakistan) in collaboration with the International Vaccine Institute, Seoul, South Korea. While the Kolkata trial showed a good effectiveness rate in all age groups, including children [17], preliminary results from Karachi differ from the Kolkata findings and do not support Vi polysaccharide vaccine protective efficacy in children 
Figure 6. Resistance patterns, S. Typhi: Kamalapur, Bangladesh (01 April 2004 - 31 March 2008)

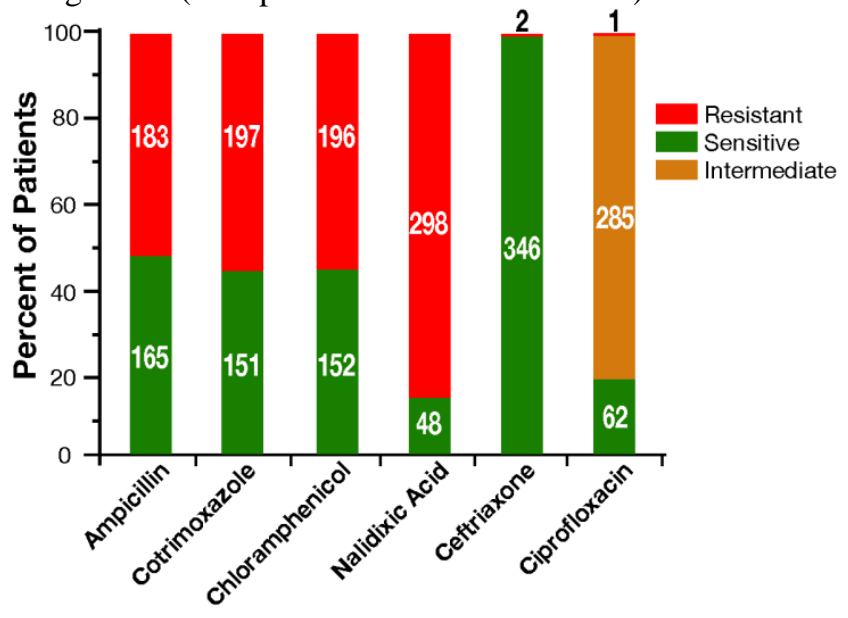

under five years following a mass vaccination program restricted to two- to 15 -year-old children (Bhutta et al., 2010 unpublished observations).

These findings, if confirmed, have potentially important implications, as they suggest that the $\mathrm{Vi}$ polysaccharide vaccine, unless used in large-scale mass vaccinations of the entire population, may not provide protection against $S$. Typhi in young children under five years of age in endemic areas. Consequently, for ensuring adequate protection of children, an alternative strategy of fast-tracking $\mathrm{Vi}$ conjugate vaccines in endemic areas may be needed.

\section{Development of novel vaccines against enteric fever}

To address the currently unmet medical need to protect infants and young children from enteric fever, a bivalent conjugate vaccine against both $S$. Typhi and $S$. Paratyphi $A$ would combine enhanced and long-lasting protection with the practical advantage of being administered at the already existing EPI vaccination visits.

NVGH is developing such a vaccine by independent chemical conjugation of the $\mathrm{Vi}$ polysaccharide of $S$. Typhi and the O polysaccharide of $S$. Paratyphi A to the carrier protein CRM197, building on Novartis's consolidated experience in conjugate vaccine development and manufacture.

NVGH envisages first the development of a monovalent $S$. Typhi vaccine, followed by the development of the bivalent conjugate vaccine also effective against $S$. Paratyphi A.

Due to the technological improvements introduced by NVGH into the manufacturing process of this vaccine, it is expected that the manufacturing yield will be high and overall costs suitable for the immunization needs of developing countries.

Preclinical data indicate that the $S$. Typhi conjugate vaccine (Vi-CRM197) is well tolerated in the animal model, and induces, even in the absence of adjuvant, an antibody response superior to that of unconjugated $\mathrm{Vi}$ polysaccharide. Vi-CRM197 is planned to enter the clinical research stage of development in 2010.

The Vi-CRM197 "first in human" clinical trial will be conducted in healthy adult volunteers in Europe, while subsequent clinical studies will be performed in collaboration with Asian scientists directly in the countries where enteric fever is endemic and the vaccine will ultimately be used. It is anticipated that the vaccine will be first licensed in Asia and subsequently prequalified by WHO.

Field trials conducted in Nepal [18] and South Africa [19] with the Vi polysaccharide vaccine and in Vietnam with the US NIH Vi conjugate vaccine [20] provide strong experimental evidence that serum antibodies to the $\mathrm{Vi}$ antigen confer protection against $S$. Typhi. Based on these data, registration and WHO pre-qualification of the NVGH conjugate vaccine will likely be based on immunogenicity data, provided that a robust and validated ELISA assay is developed and that the immunogenicity of ViCRM197 in infants is adequate.

\section{Strategies for introduction of novel vaccines against enteric fever}

The preferred age for immunization with the conjugate vaccines was also discussed. The meeting participants suggested that the new $\mathrm{Vi}$ conjugate vaccine could be administered either to young infants, as 3 doses alongside the current EPI schedule at 6,10 and 14 weeks of age (the optimal schedule to achieve a high coverage and avoid costs of additional immunization visits), or to older infants, concomitantly with measles immunization at 9 to 12 months. Particularly with older infants, the challenge will be to ensure adequate coverage given that a booster dose would likely be needed. Although the booster dose could potentially be given at 18 months of age with a fourth dose of DTP vaccine, it should be considered that the current coverage for the DTP booster is only about $20 \%$.

Both approaches offer advantages and disadvantages, and the preferred age group for administration of conjugate vaccines against enteric fever will have to be selected based on clinical and 
Table 1. Licensed vaccines against $S$. Typhi *

\begin{tabular}{|c|c|c|}
\hline Vaccine type & Live attenuated & Unconjugated Vi \\
\hline Vaccine [Manufacturer] & [Crucell | Berna] & $\begin{array}{l}\text { [GSK; Sanofi-Pasteur; Bharat, Finlay, } \\
\text { IVAC, Shantha etc.] }\end{array}$ \\
\hline Target age group & $>6$ years of age & $>2$ years of age \\
\hline Number of doses & $\begin{array}{l}\text { multiple doses } \\
\text { revaccination every } 5 \text { years }\end{array}$ & $\begin{array}{l}1 \text { dose } \\
\text { revaccination every } 2 \text { years }\end{array}$ \\
\hline Route of delivery & Oral, enteric coated capsules & Parenteral \\
\hline Efficacy & $50-80 \%$ & $64-72 \%$ \\
\hline Duration of protection & At least 5 years & At least 3 years \\
\hline Protective immunity & $\begin{array}{l}\text { CMI } \\
\text { Antibody (non-Vi) }\end{array}$ & Anti-Vi antibodies \\
\hline
\end{tabular}

* No licensed vaccines are available against $S$. Paratyphi A

epidemiological considerations, as well as costeffectiveness and policy concerns that are not entirely available at the moment but should be addressed.

The value of developing combination vaccines by formulating Salmonella antigens together with EPI vaccines was also discussed. While this approach would reduce the number of injections and of adverse events and wastage, technical and programmatic problems may limit the number and composition of different combinations. The participants agreed that even if appropriate combinations are developed, stand-alone enteric fever vaccines will be needed to have more flexibility to immunize different age groups and for implementation of mass immunization and catch-up campaigns.

The benefits of introducing such a novel vaccine against enteric fever would be considerable for endemic countries. However, in order to successfully introduce any novel vaccine into the national immunization plans, as shown by previous experiences with other vaccines (e.g., Hib and pentavalent vaccines), not only credible and supportive clinical, epidemiological and health economic data should be generated, but, in addition, these data must be appropriately presented to policymakers and endorsed by them.

When asked to identify gaps in the data available, a few messages emerged from the experts in the audience regarding epidemiology. While data from urban areas (Kolkata, Delhi, Karachi, and Dhaka) is available, further data needs to be collected from rural settings in order to have a more complete picture of the epidemiology of the region. Mortality is not often observed in active surveillance studies, but other measures of morbidity should be better characterized, including the financial cost of severe cases, (e.g., multi-drug resistance, hospitalizations, etc.). More studies are needed to better understand the potential savings associated with a decrease in severe cases and hospital utilization. Finally, since typhoid fever immunization is currently not perceived as a high priority in the community compared to other immunizations, more work should be done to raise awareness of the need for vaccination against typhoid fever.

\section{Overall discussion and conclusions}

The meeting provided the participants with a consolidated view of the available data, both published and unpublished, confirming the high burden of typhoid and paratyphoid fever in urban areas of Bangladesh, India, and Pakistan, including in children under two years of age. Based on limited data, it is estimated that rural areas are less affected, although rapid urbanization might change this epidemiological scenario.

Improvements in living conditions (better hygiene, sanitation, piped water, etc.) would undoubtedly improve the situation, but will take 
many years to be implemented. On these grounds, all the participants emphasized that the rapid introduction of effective and affordable enteric fever vaccines into the EPI pediatric programs of at least the urban slums of Bangladesh, India, and Pakistan should be seriously considered. Both $S$. Typhi and $S$. Paratyphi $A$ need to be addressed and therefore a bivalent conjugate vaccine would be highly desirable. However, considering the prevalence of $S$. Typhi in South Asia and the advanced stage of development of the $\mathrm{Vi}$ polysaccharide conjugate vaccine, the participants felt that countries need to consider the introduction of an $S$. Typhi conjugate vaccine in their national immunization programs as soon as possible. At the same time, active epidemiological surveillance should be continued to allow an evidence-based decision to replace the monovalent $S$. Typhi vaccine with the bivalent $\mathrm{S}$. Typhi/S. Paratyphi vaccine, as soon as it becomes available for use.

\section{References}

1. World Health Organization (2008) The global burden of disease: $2004 \quad$ update. Available:http://www.who.int/healthinfo/global burden disease/ 2004 report_update/en/index.html Accessed 27 February 2010.

2. Saul A, Rappuoli R (2008) The Novartis Vaccines Institute for Global Health: a new initiative for developing vaccines for neglected diseases in developing countries. J Infect Developing Countries 2: 154-155.

3. Podda A (2010) Aims and role of Novartis Vaccines Institute for Global Health (NVGH): Procedia in Vaccinology "Proceedings of the Ninth Global Vaccine Research Forum and Parallel Satellite Symposia, Bamako, Mali, 6-9, December 2009. In press.

4. Crump JA, Luby SP, Mintz ED (2004) The global burden of typhoid fever. Bull World Health Organ 82: 346-353.

5. Panikar CKJ. Vimala KN. (1092) Transferable chloramphenicol resistance in S. Typhi. Nature 239: 109110.

6. Wain J and Kidgell C (2004) The emergence of multidrug resistance to antimicrobial agents for the treatment of typhoid fever. Trans R Soc Trop Med Hyg 98: 423-430.

7. Guzman CA, Borsutzky S, Griot-Wenk M, Metcalfe IC, Pearman J Collioud A, Favre D, Dietrich G (2006) Vaccines against typhoid fever. Vaccine 24: 3804-3811.

8. World Health Organization (2008) Meeting of the immunization Strategic Advisory Group of Experts, November 2007: conclusions and recommendations. WER 1: $1-16$.

9. Patnaik KC and Kapoor PN (1967) A note on incidence of typhoid in Delhi. Indian J Med Res 55: 3.

10. Central Bureau of Health Intelligence (1988) Reported Cases and deaths due to communicable diseases in India during 1987. In: Health Information India. New Delhi, India: Ministry of Health and Family Welfare, Govt. of
India, New Delhi. p.177-79.

11. Central Bureau of Health Intelligence (1994) Reported Cases and deaths due to communicable diseases during 1994. In: Health Information India. New Delhi, India: Ministry of Health and Family Welfare, Govt. of India, New Delhi. p. 185-87.

12. Sinha A, Sazawal S, Kumar R, Sood S, Reddaiah VP, Singh B, Rao M, Naficy A, Clemens JD, Bhan MK (1999) Typhoid fever in children aged less than 5 years. Lancet 354: 734-37.

13. Ochiai RL, Acosta CJ, Danovaro-Holliday MC, Baiqing D, Bhattacharya SK, Agtini MD, Bhutta ZA, Canh DG, Ali M, Shin S, Wain J, Page AL, Albert MJ, Farrar J, Abu-Elyazeed R, Pang T, Galindo CM, von Seidlein L, Clemens JD, and the Domi Typhoid Study Group (2008) A study of typhoid fever in five Asian countries: disease burden and implications for controls. Bull World Health Organ 86: 26068.

14. Kumar R, Sazawal S, Sinha A, Sood S, Bhan MK (1997) Typhoid fever: contemporary issues as related to the disease in India. Round Table Conference Series on Water Borne Diseases. 12th ed. Ranbaxy Science Foundation, New Delhi, 2: $31-36$

15. Sood S, Kapil A, Dash N, Das BK, Goel V, Seth P (1999) Paratyphoid fever in India: An emerging problem. Emerging infectious diseases 5:483-484

16. Walia M, Gaind R, Mehta R, Paul P, Aggarwal P, Kalaivani M (2005) Current perspectives of enteric fever: a hospitalbased study from India. Annals of Tropical Paediatrics: International Child Health, 25: 161-174.

17. Sur D, Ochiai RL, Bhattacharya SK, Ganguly NK, Ali M, Manna B, Dutta S, Donner A, Kanungo S, Park JK, Puri MK, Kim DR, Dutta D, Bhaduri, Acosta CJ, Clemens JD (2009) A cluster-randomized effectiveness trial of Vi typhoid vaccine in India. N Engl J Med 361: 335-44.

18. Acharya IL, Lowe CU, Thapa R, Gurubacharya VL, Shrestha MB, Cadoz M, (1987) Prevention of typhoid fever in Nepal with the Vi capsular polysaccharide of Salmonella Typhi NEJM 317: 1101-1104.

19. Klugman KP, Koornhor HJ, Robbins JB, Le Cams NN (1996) Immunogenicity, efficacy and serological correlate of protection of Salmonella Typhi Vi capsular polysaccharide vaccine three years after immunization. Vaccine 14: 435-43.

20. Lin FYC, Ho VA, Khiem HB, Trach DD, Bay PV, Thanh TC, Kossaczka Z, Bryla DA, Shiloach J, Robbins JB, Schneerson R, Szu SC (2001) The efficacy of a Salmonella Typhi Vi conjugate vaccine in two-to-five-year-old children. NEJM 344:1263-1269.

\section{Corresponding author}

Audino Podda, MD

Novartis Vaccines Institute for Global Health

Via Fiorentina, 1 - 53100 Siena, Italy

Phone: +390577 243496

Fax: +390577243540

Email: audino.podda@novartis.com

Conflict of interests: No conflict of interests is declared. 